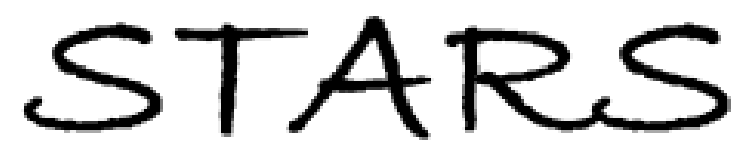

University of Central Florida

STARS

Faculty Bibliography 2010s

Faculty Bibliography

$1-1-2011$

\title{
Infrared surface plasmons on heavily doped silicon
}

Monas Shahsad

University of Central Florida

Gautam Medhi

University of Central Florida

Robert E. Peale

University of Central Florida

Walter R. Buchwald

Justin W. Cleary

See next page for additional authors

Find similar works at: https://stars.library.ucf.edu/facultybib2010

University of Central Florida Libraries http://library.ucf.edu

This Article is brought to you for free and open access by the Faculty Bibliography at STARS. It has been accepted for inclusion in Faculty Bibliography 2010 s by an authorized administrator of STARS. For more information, please contactSTARS@ucf.edu.

\section{Recommended Citation}

Shahsad, Monas; Medhi, Gautam; Peale, Robert E.; Buchwald, Walter R.; Cleary, Justin W.; Soref, Richard; Boreman, Glenn D.; and Edwards, Oliver, "Infrared surface plasmons on heavily doped silicon" (2011).

Faculty Bibliography 2010s. 1890.

https://stars.library.ucf.edu/facultybib2010/1890

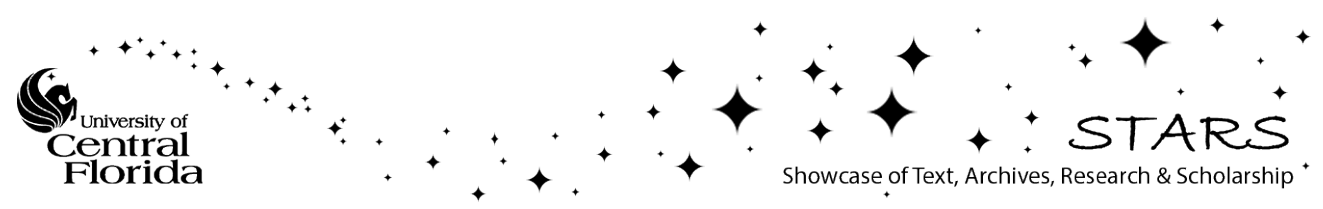




\section{Authors}

Monas Shahsad, Gautam Medhi, Robert E. Peale, Walter R. Buchwald, Justin W. Cleary, Richard Soref, Glenn D. Boreman, and Oliver Edwards 


\section{Infrared surface plasmons on heavily doped silicon}

Cite as: J. Appl. Phys. 110, 123105 (2011); https://doi.org/10.1063/1.3672738

Submitted: 06 October 2011 . Accepted: 29 November 2011 . Published Online: 27 December 2011

Monas Shahzad, Gautam Medhi, Robert E. Peale, Walter R. Buchwald, Justin W. Cleary, Richard Soref, Glenn D. Boreman, and Oliver Edwards

\section{ARTICLES YOU MAY BE INTERESTED IN}

Infrared plasmons on heavily-doped silicon

Journal of Applied Physics 110, 043110 (2011); https://doi.org/10.1063/1.3626050

Epitaxial growth of engineered metals for mid-infrared plasmonics

Journal of Vacuum Science \& Technology B 31, 03C121 (2013); https://doi.org/10.1116/1.4797487

Localized surface plasmon resonances in highly doped semiconductors nanostructures

Applied Physics Letters 101, 161113 (2012); https://doi.org/10.1063/1.4760281

\section{Applied Physics Reviews} Now accepting original research

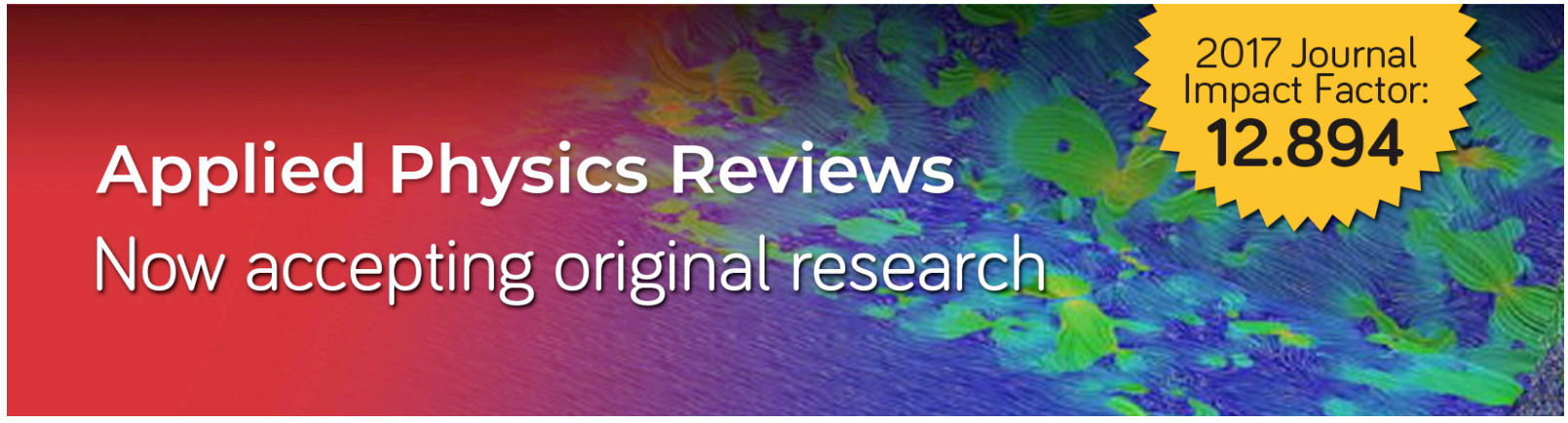




\title{
Infrared surface plasmons on heavily doped silicon
}

\author{
Monas Shahzad, ${ }^{1}$ Gautam Medhi, ${ }^{1}$ Robert E. Peale, ${ }^{1, a)}$ Walter R. Buchwald, ${ }^{2}$ \\ Justin W. Cleary, ${ }^{3}$ Richard Soref, ${ }^{4}$ Glenn D. Boreman, ${ }^{5}$ and Oliver Edwards ${ }^{6}$ \\ ${ }^{1}$ Department of Physics, University of Central Florida, Orlando, Florida 32816, USA \\ ${ }^{2}$ Solid State Scientific Corporation, 27-2 Wright Road, Hollis, New Hampshire 03049, USA \\ ${ }^{3}$ Sensors Directorate, Air Force Research Laboratory, Wright-Patterson Air Force Base, Ohio 45433, USA \\ ${ }^{4}$ Department of Physics, University of Massachusetts, Boston, Massachusetts 02125, USA \\ ${ }^{5}$ Department of Physics and Optical Science, University of North Carolina at Charlotte, Charlotte, \\ North Carolina 28223, USA \\ ${ }^{6}$ Zyberwear Inc., 2650 Florence St., Orlando, Florida 34818, USA
}

(Received 6 October 2011; accepted 29 November 2011; published online 27 December 2011)

\begin{abstract}
Conductors with infrared plasma frequencies are potentially useful hosts of surface plasmon polaritons (SPP) with sub-wavelength mode confinement for sensing applications. A challenge is to identify such a conductor that also has sharp SPP excitation resonances and the capability to be functionalized for biosensor applications. In this paper we present experimental and theoretical investigations of IR SPPs on doped silicon and their excitation resonances on doped-silicon gratings. The measured complex permittivity spectra for $\mathrm{p}$-type silicon with carrier concentration $6 \times 10^{19}$ and $1 \times 10^{20} \mathrm{~cm}^{-3}$ show that these materials should support SPPs beyond 11 and $6 \mu \mathrm{m}$ wavelengths, respectively. The permittivity spectra were used to calculate SPP mode heights above the silicon surface and SPP propagation lengths. Reasonable merit criteria applied to these quantities suggest that only the heaviest doped material has sensor potential, and then mainly within the wavelength range 6 to $10 \mu \mathrm{m}$. Photon-to-plasmon coupling resonances, a necessary condition for sensing, were demonstrated near $10 \mu \mathrm{m}$ wavelength for this material. The shape and position of these resonances agree well with simple analytic calculations based on the theory of Hessel and Oliner (1965). (C) 2011 American Institute of Physics. [doi:10.1063/1.3672738]
\end{abstract}

\section{INTRODUCTION}

Bound electromagnetic waves that propagate along the interface between a dielectric and a conductor exist below the plasma frequency of the conducting material when the real part of the permittivity $\varepsilon^{\prime}$ is negative. ${ }^{1}$ Tight confinement of SPP energy to distances above the interface less than the corresponding free-space wavelength requires frequencies not too far below the plasma frequency, which holds at optical wavelengths for metals like gold and silver.

The principal existing application of surface plasmon polaritons (SPPs) is in biosensors where the change in the resonant coupling of light to SPPs indicates the binding of biomolecules to the conductor surface. ${ }^{2}$ Commercial Surface Plasmon Resonance (SPR) biosensors work at visible or near infrared frequencies. ${ }^{3,4}$ It has been proposed that operating SPR biosensors in the mid-IR region may yield increased sensitivity and selectivity because the biological analytes are then differentiated by their IR "fingerprint" absorption spectra, and analyte refractive index changes are enhanced by the associated dispersion. ${ }^{5}$ The long wavelength limit of one of the few previous IR SPR biosensor investigations ${ }^{3}$ was 2.3 $\mu \mathrm{m}$, which falls short of the range of characteristic molecular absorption lines. It has also been suggested ${ }^{6}$ that these longer wavelengths are less likely to cause photo-induced damage to biological specimens, but the longest wavelength studied in this work was $2 \mu \mathrm{m}$, well short of the molecular finger-

a)Electronic mail: robert.peale@ucf.edu. print region. In contrast, the experimental SPR results we present here are at $10 \mu \mathrm{m}$ wavelengths.

Optimal overlap and interaction between IR SPPs and surface-bound analytes requires conductors with IR plasma frequencies. ${ }^{7}$ One possibility is heavily doped silicon, whose long wave electrodynamic properties at carrier concentrations above $6 \times 10^{19} \mathrm{~cm}^{-3}$ are those of a conductor that can support SPPs. ${ }^{7-14}$ Doped Si as a mid-IR SPP host for SPR biosensors was suggested independently by Cleary et $\mathrm{al}^{7}$ and by Chen et al. ${ }^{11}$ The latter theoretically studied SPR on silicon gratings. A modified Si-based design was proposed and studied theoretically by DiPippo et al. ${ }^{12}$ Recently, Ginn et al. ${ }^{13}$ experimentally determined the optical constants for a range of ion-implanted silicon samples and predicted their long-wave IR SPP properties. They wrote, "The direct measurement of surface plasmon propagation can be experimentally quantified using standard prism coupling and grating scattering techniques." Preliminary experiments of SPPs on doped silicon by prism coupling ${ }^{5}$ and grating scattering ${ }^{14}$ has already been presented in conference proceedings. This paper presents a more extended experimental investigation of heavily-doped silicon as an IR SPP host material and reports measured SPR effects on Si gratings.

Silicon is also interesting for $\mathrm{p}^{+}-\mathrm{Si} / \mathrm{Si}$ hybrid plasmonic and photonic waveguiding applications, ${ }^{8}$ which could allow for the integration of multiple functions on a single chip fabricated using standard silicon processing. Narrow subwavelength guiding regions of any desired shape and doping density are readily created by ion implantation through a photolithographic mask. SPPs are localized near the conductive, 
doped surface, which would act as a useful "interface" for analytes in advanced chem-bio plasmonic sensing architectures. SPPs excited by photons at the doped-waveguide input and sensed via photonic coupling at the output could be utilized for spectrometer-on-a-chip applications. For example, $\mathrm{Si}$ plasmo-photonic waveguide coupling may be achieved via two Si strip channel photonic waveguides on a silicon-on-insulator substrate that are each coupled via a silicon taper to a doped-Si on $\mathrm{Si}$ plasmonic waveguide whose cross section is below the cut-off frequency at the operational wavelength. In addition to its photonic compatibility, the potential advantages of silicon plasmonics are its CMOS compatibility, its manufacturability in a foundry, and its ability to be biologically functionalized.

Small discrete plasmonic devices operate successfully in the near infrared and visible. However, if we consider "elongated" devices that serve as plasmonic channel waveguides, then the SPP propagation loss becomes a figure of merit. A useful criterion is that the characteristic propagation length for SPP intensity should be at least twice the freespace wavelength. ${ }^{8}$ This consideration favors long IR wavelengths, because the loss drops rapidly as the frequency is decreased below the plasma frequency $\omega_{\mathrm{p}}$. On the other hand, for biosensor applications, the mode should strongly overlap the surface bound analyte and hence should be tightly confined to the surface. A reasonable criterion is that the characteristic penetration depth for electric field into the space above the conductor should be less than the free space wavelength. ${ }^{8}$ However, the SPP mode height is sub-wavelength only near $\omega_{\mathrm{p}}$, and it increases rapidly for longer wavelengths. Thus, these two figures of merit with conflicting spectral requirements must be balanced and optimized.

The IR photonic/plasmonic waveguide suggested for illustration is a composite of conductors (doped $\mathrm{Si}$ ) and dielectrics (undoped $\mathrm{Si}$ ). The plasma frequency $\omega_{\mathrm{p}}$ of the conducting regions is determined by the carrier concentration and carrier effective mass. In the undoped regions, the material should be transparent, which is generally satisfied in the IR for semiconductors.

While the preceding discussion presents a vision for a $\mathrm{Si}$ biosensor on micron length scales, actual commercial biosensors couple light to SPPs by illuminating a thin conducting layer through a bulk prism. The prism allows matching of photon and SPP momenta so that coupling can occur at the appropriate angle of incidence. Successful coupling is observed as a resonant decrease in the specularly reflected power. Infrared materials, and in particular $\mathrm{Si}$, have high refractive indices which leads to strong interference artifacts in the angular reflection spectrum associated with hemicylindrical prisms and to impractically large apex angles for triangular prisms. ${ }^{5}$ These problems associated with SPR biosensors at IR wavelengths motivate the alternative to prism couplers, namely grating couplers, ${ }^{11-17}$ which is the approach adopted in this experimental study of SPR excitations on doped silicon.

\section{EXPERIMENTAL DETAILS}

Commercial p-Si wafers with resistivities $\rho=0.0006$ 0.001 and $0.0018-0.0032 \Omega-\mathrm{cm}$ were procured. These were boron-doped during the melt with uniform carrier concentration throughout. Resistivity values were confirmed by standard 4-pt probe measurements, and the carrier concentrations, corresponding to our measured values for the samples studied, are $1 \times 10^{20} \mathrm{~cm}^{-3}$ and $6 \times 10^{19} \mathrm{~cm}^{-3}$, respectively. ${ }^{18}$ Complex permittivity spectra of the unprocessed wafers were determined using a Woollam IR-VASE ellipsometer in the wavelength range 1 to $40 \mu \mathrm{m}$. Lamellar surface-corrugation gratings of $20 \mu \mathrm{m}$ period, nominal $50 \%$ duty cycle, and various amplitudes (i.e., etch depth) were formed by standard photolithography and reactive ion etching. Profiles were confirmed using a step profilometer.

Figure 1 presents a schematic diagram of the experimental setup for measuring the infrared specular reflection as a function of angle, where ' $\mathrm{L}$,' ' $\mathrm{D}$,' 'G,' and 'GR' in the diagram represent laser, detector, goniometer, and grating. A ppolarized line-tunable $\mathrm{CO}_{2}$ laser or quantum-cascade laser (QCL) were used together with a laser power meter or a $\mathrm{HgCdTe}$ photodetector operated at $77 \mathrm{~K}$, respectively. Dips in the reflected power at certain angles of incidence indicate the excitation of SPPs. ${ }^{16}$

\section{THEORETICAL CONSIDERATIONS}

IR-to-SPP grating couplers function by adding positive and negative integer multiples $m$ of $2 \pi / p$ to the in-plane component of the photon wave vector, where $p$ is the grating period. ${ }^{15-17}$ This compensates for the inherent photon/SPP momentum mismatch. Grating couplers allow multiple SPP excitation resonances due to multiple units of grating momentum that may be added, if higher harmonics of the grating period occur in its profile. ${ }^{15}$ The coupling condition between an IR wave, which is incident from a dielectric at an angle $\theta$ onto a conducting grating of period $p$, and an SPP is

$$
\eta_{d} \sin (\theta)+m \frac{\lambda}{p}= \pm \frac{c}{\omega} \operatorname{Re}\left[k_{\mathrm{SPP}}\right] .
$$

The refractive index $\eta_{d}$ of the dielectric above the grating is important in biosensing applications, since it changes when

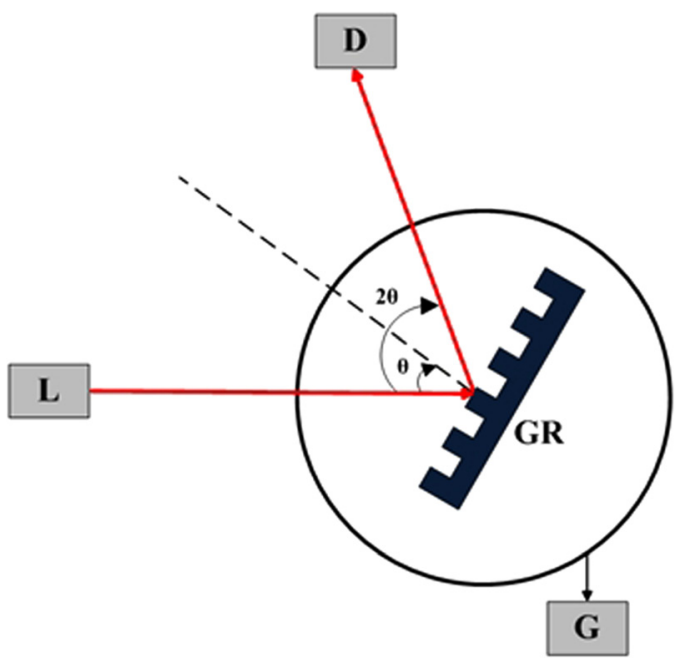

FIG. 1. (Color online) Schematic diagram of experimental setup for measuring the infrared specular reflection as a function of angle, where labels L, $\mathrm{D}, \mathrm{G}$, and GR in diagram represent laser, detector, goniometer, and grating. 
analyte molecules bind to the surface and shift the resonance angles. In equation (1), the complex SPP wave vector $k_{\mathrm{spp}}$ is ${ }^{16}$

$$
k_{S P P}(\omega)=\frac{\omega}{c} \sqrt{\frac{\varepsilon_{d} \varepsilon_{c}}{\varepsilon_{d}+\varepsilon_{c}}},
$$

where $c$ is the light speed, $\varepsilon_{d}$ and $\varepsilon_{c}$ the complex permittivity of dielectric and conductor, respectively, and $\omega$ the angular frequency. The complex permittivity spectrum $\varepsilon_{\mathrm{c}}(\omega)=\varepsilon_{c}{ }^{\prime}$ $(\omega)+i \varepsilon_{\mathrm{c}}^{\prime \prime}(\omega)$ was determined from the measured IR ellipsometry spectra of our p-Si wafers using standard Fresnel equations, ${ }^{19}$ and values at our laser wavelengths are presented in Table I. For the measurements presented here, $\varepsilon_{d}=\eta_{d}=1$, and the subscript " $c$ " in the conductor permittivity will be dropped in the subsequent discussion.

Calculation of angular reflection spectra was performed using the theory of Hessel and Oliner. ${ }^{15-17}$ This theory allows analytic calculation of the line shapes for absorption resonances that are associated with the generation of "guided complex waves supportable by the grating." Though the words "surface plasmon polariton" do not appear in this early paper, ${ }^{17}$ the wave function and complex wavevector of the guided waves are identical to those of SPPs, so they are the same thing. Moreover, propagating SPPs associated with measured resonances that agree with such calculations have been directly observed. ${ }^{15}$

We assume a simplified model of the grating as sinusoidally modulated surface impedance, represented by

$$
Z(x)=Z_{0}\left(1+M \cos \left[\frac{2 \pi x}{p}\right]\right)
$$

where $x$ is the coordinate in the plane of the grating and perpendicular to the grooves, and $M$ is a modulation parameter that is a function of grating permittivity, amplitude, and wavelength. This approximation neglects the higher order Fourier components of the actual rectangular grating profile. $Z_{0}$, the average surface impedance, or the impedance in case of zero modulation is

$$
Z_{0}=\sqrt{\frac{\mu_{0}}{\left(\varepsilon^{\prime}+i \varepsilon^{\prime \prime}\right) \varepsilon_{0}}}=\frac{377 \Omega}{\sqrt{\varepsilon^{\prime}+i \varepsilon^{\prime \prime}}},
$$

where $\mu_{0}, \varepsilon_{0}$, and $377 \Omega$ are, respectively, the permeability, permittivity, and impedance of free space. The average surface impedance relative to free space is

$$
\zeta \equiv \frac{Z_{0}}{377 \Omega}=\frac{1}{\sqrt{\varepsilon^{\prime}+i \varepsilon^{\prime \prime}}}
$$

TABLE I. Optical parameters of heavily doped p-type Si.

\begin{tabular}{lrccc}
\hline \hline $\mathrm{N}\left(\times 10^{19} \mathrm{~cm}^{-3}\right)$ & $\lambda(\mu \mathrm{m})$ & $\varepsilon^{\prime}$ & $\varepsilon^{\prime \prime}$ & $\zeta$ \\
\hline 10 & 9.25 & -11.4139 & 15.6130 & $0.1029-i 0.2028$ \\
6 & 9.38 & 2.76364 & 4.80317 & $0.3677-i 0.2126$ \\
10 & 10.59 & -16.1949 & 20.9584 & $0.0856-i 0.1744$ \\
\hline \hline
\end{tabular}

or

$$
\zeta=\left[\begin{array}{c}
-i \\
1
\end{array}\right]\left(\varepsilon^{2}+\varepsilon^{\prime \prime 2}\right)^{-1 / 4}\left\{\cos \left(\frac{\varphi}{2}\right)-i \sin \left(\frac{\varphi}{2}\right)\right\} \text { for } \begin{aligned}
& \varepsilon^{\prime}<0 \\
& \varepsilon^{\prime}>0
\end{aligned}
$$

where $\varphi=\tan ^{-1}\left(\varepsilon^{\prime \prime} / \varepsilon^{\prime}\right)$. Equation (6) has four mathematically possible pre-factors $( \pm 1, \pm i){ }^{20}$ On physical grounds, ${ }^{21}$ we require $\zeta^{\prime}>0$ and $\zeta^{\prime \prime}<0$, which leads to just two pre-factors being retained, with each pertaining to particular permittivity conditions as shown in Eq. (6). The specular reflectance of the grating is calculated to good approximation by

$$
R \approx\left|1-\frac{4 \cos \theta}{M \zeta\left(D_{0}-D_{1}^{-1}-D_{-1}^{-1}\right)}\right|^{2}
$$

with $D_{n}$ defined as

$$
D_{n}=\frac{2}{M}\left[1+\frac{1}{\zeta} \sqrt{1-\left(\sin \theta+\frac{n \lambda}{p \eta_{d}}\right)^{2}}\right]
$$

with $n$ an integer. The sinusoidal surface impedance approximation and the neglect of $D_{n}$ terms with $|n|>1$ in the formula for $R$ give calculated angular reflectance spectra in which only the $m=1$ resonance appears. Inclusion of higher order $D_{n}$ terms in the formula for $R$ has negligible effect on the reflectance spectrum in the angular regions accessible to our experiments. For fitting the measured angular reflectance spectra, $M$ is the only parameter varied.

The 1/e penetration depth of the electric field into the air above the conductor is given by

$$
L_{d}=\left[\frac{\omega}{c} \operatorname{Re} \sqrt{\frac{-1}{1+\varepsilon}}\right]^{-1},
$$

while the SPP intensity decay length in the direction of propagation is given by ${ }^{16}$

$$
L_{z}=\frac{1}{2 \operatorname{Im}\left[k_{S P P}\right]} .
$$

\section{RESULTS}

Figure 2 presents permittivity spectra for both wafers. For the most heavily doped silicon, the $\varepsilon^{\prime}$ values are negative below $0.212 \mathrm{eV}$ (wavelength $\lambda>5.84 \mu \mathrm{m}$ ). The imaginary part exceeds the magnitude of the real part, which is generally disadvantageous for SPP applications as $\varepsilon^{\prime \prime}$ is responsible for loss and SPR broadening. For the more lightly doped silicon, the $\varepsilon^{\prime}$ values are negative below $0.107 \mathrm{eV}(\lambda>11.6$ $\mu \mathrm{m})$. Here also $\varepsilon^{\prime \prime}$ exceeds the magnitude of $\varepsilon^{\prime}$. At short wavelengths the real part of the permittivity for both materials approaches 11.3, which is close to the static dielectric constant value of 11.7 , while the imaginary parts approach zero. The permittivity values for the doped $\mathrm{p}-\mathrm{Si}$, relevant to the experiments performed using $\mathrm{CO}_{2}$ and quantum-cascade 


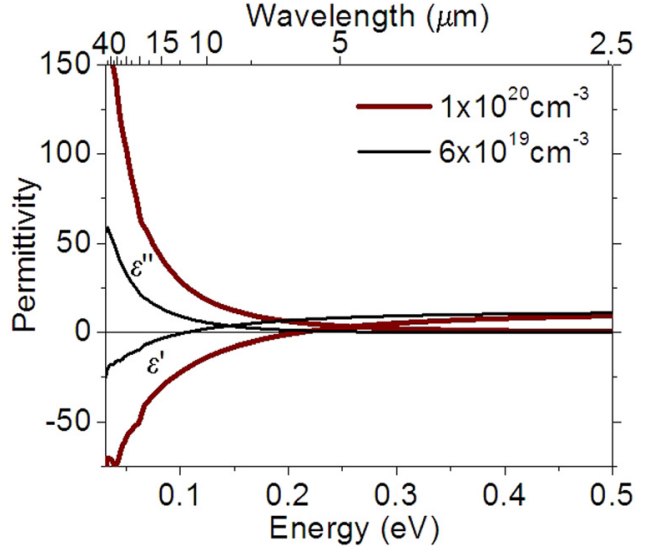

FIG. 2. (Color online) Real and imaginary parts of the permittivity for heavily-doped p-type silicon of different carrier concentration, as indicated in the legend.

lasers, are given in Table I, together with the relative surface impedance.

Figure 3 presents the experimental reflected intensity as a function of angle of incidence at two different $\mathrm{CO}_{2}$ laser wavelengths for gratings of different amplitude $h$ made from the $1 \times 10^{20} \mathrm{~cm}^{-3}$ silicon. The data have been normalized, so that the maximum experimental reflected power has the value unity, and the curves for increasing $h$ have been offset vertically from each other by unity for clarity. For $h=0.45$ $\mu \mathrm{m}$, absorption resonances are just discernable for the 10.59 $\mu \mathrm{m}$ wavelength, while at $9.25 \mu \mathrm{m}$ wavelength they are partly obscured by baseline variations caused by slow changes in the $\mathrm{CO}_{2}$ laser output power. In general, the resonances strengthen up to about $h=1 \mu \mathrm{m}$, beyond which the resonances broaden, the $m=1$ resonances become more shallow, and the $m=-3$ resonances continue to deepen. The resonances are rather broad for all grating amplitudes, as was anticipated from the large $\varepsilon^{\prime \prime}$ values. The $m=1$ resonance, according to Eq. (1), occurs at 34.45 and $29.60 \mathrm{deg}$ for $\lambda=9.250$ and $10.591 \mu \mathrm{m}$, respectively. These angles are indicated by symbols and the labels "1" in Fig. 3. The $m=-3$ resonance occurs at 21.53 and 34.40 deg for the two

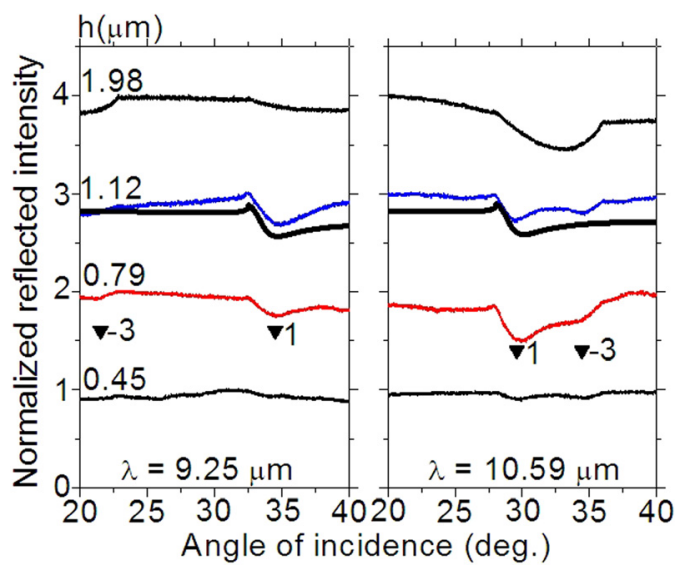

FIG. 3. (Color online) Experimental angular reflectance spectra for $\mathrm{p}-\mathrm{Si}$ with carrier concentration of $1 \times 10^{20} \mathrm{~cm}^{-3}$ fabricated into lamellar gratings of $20 \mu \mathrm{m}$ period and with various amplitudes $h$ as indicated. The heavy black curves for $h=1.12 \mu \mathrm{m}$ are calculated spectra. respective wavelengths. These angles are indicated by symbols labeled "- 3" in Fig. 3. Angles from Eq. (1) are in good agreement with the observed reflectivity dips. No other resonances, besides the $m=1$ and -3 ones, are predicted to occur in the angular range of Fig. 3. Calculated resonance spectra are also plotted in Fig. 3 as heavy black lines for $h=1.12 \mu \mathrm{m}$, where the best match was found using $M=1 \mathrm{in}$ Eqs. $(7,8)$. These calculated spectra closely resemble the experimental ones, except that as mentioned above, only the $m=1$ resonance appears in the calculated curve because of the assumed sinusoidal impedance modulation with no higher harmonics.

Figure 4 presents the experimental reflected intensity (thin curve, normalized) as a function of angle of incidence at the QCL wavelength of $9.38 \mu \mathrm{m}$ for the silicon gratings of carrier concentration $6 \times 10^{19} \mathrm{~cm}^{-3}$ and amplitude $h=1 \mu \mathrm{m}$. Only a baseline drift without suggestion of a resonance is observed. The cusp at $\sim 34 \mathrm{deg}$ in the calculated resonance spectrum (heavy curve, un-normalized) with $M=1$ in Eq. (8) suggests a hint of a resonance. The absence of a clear resonance excitation of an SPP is expected as no SPP should be supported at this wavelength for this material, since its permittivity is positive here (Table I).

Figure 5 presents the SPP field penetration depth $L_{d}$ above the conductor surface calculated from Eq. (9) using the measured permittivity spectra. For carrier concentration $1 \times 10^{20} \mathrm{~cm}^{-3}$ and $\lambda>5.8 \mu \mathrm{m}$, we have $\varepsilon^{\prime}<0$, so here this material supports a traditional bound SPP. For $\lambda<5.8 \mu \mathrm{m}$, $\varepsilon^{\prime}>0$ and $\varepsilon^{\prime \prime}$ approaches zero, so there the Si behaves like a dielectric: No bound SPP is supported and $L_{d}$ quickly becomes $\gg \lambda$. For carrier concentration $6 \times 10^{19} \mathrm{~cm}^{-3}$, the behavior is similar, but shifted to longer wavelength. Our criterion for application to a useful SPP-based sensor is $L_{d}<\lambda$. For carrier concentration $1 \times 10^{20} \mathrm{~cm}^{-3}$ this holds for $5.1<\lambda<9.8 \mu \mathrm{m}$. For carrier concentration $6 \times 10^{19} \mathrm{~cm}^{-3}$, it holds for $9.6<\lambda<16.6 \mu \mathrm{m}$. By comparison, the sub wavelength confinement for doped silicon calculated by Ginn et $a l .{ }^{13}$ at p-type concentration of $10^{20} \mathrm{~cm}^{-3}$ occurs at somewhat longer wavelength, namely, from 7 to 12 microns. The difference is within the range of uncertainty for estimating the carrier concentration from the measured resistivity.

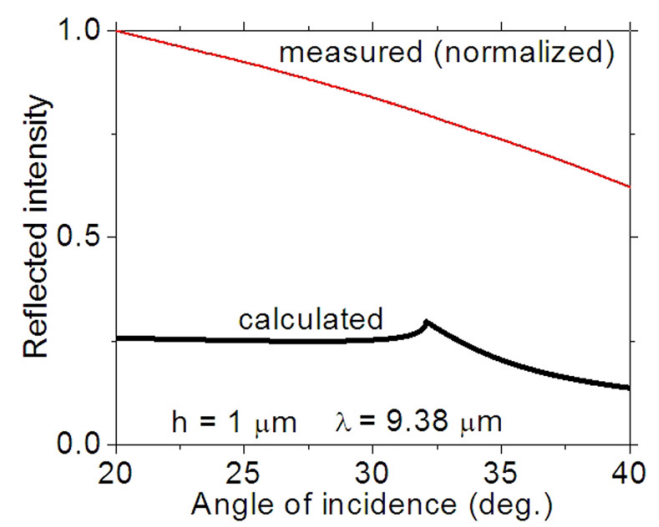

FIG. 4. (Color online) Experimental (thin curve, normalized) and calculated (heavy curve, un-normalized) angular reflectance spectra at $9.38 \mu \mathrm{m}$ wavelength excitation for the $\mathrm{p}^{+}-\mathrm{Si}$ with concentration $6 \times 10^{19} \mathrm{~cm}^{-3}$. The lamellar grating had a $20 \mu \mathrm{m}$ period with $1 \mu \mathrm{m}$ amplitude. 


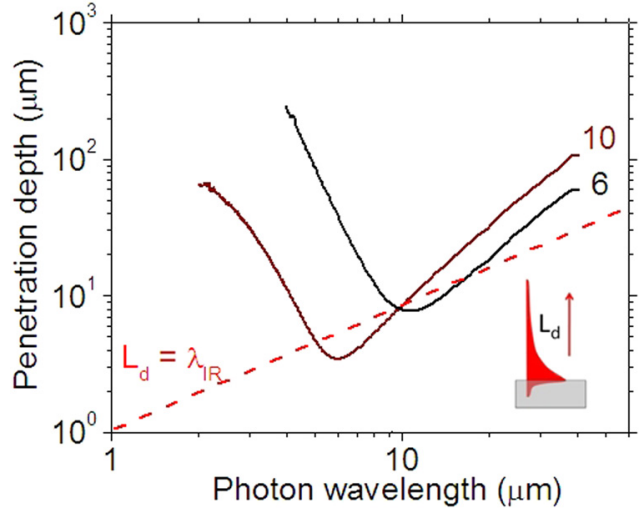

FIG. 5. (Color online) SPP field penetration depth into vacuum above the $\mathrm{p}^{+}$-Si surface. The part of the curves that fall below the dotted straight line $\left(L_{d}=\lambda\right)$ are where the SPP electric field confinement is less than the free space wavelength. The numbers that label the curves give the carrier concentrations $\left(\times 10^{19} \mathrm{~cm}^{-3}\right)$. The inset is a schematic to suggest the exponential decay of the SPP field away from the interface and the significance of $L_{d}$.

Figure 6 presents the characteristic SPP energy propagation length $L_{z}$ as a function of free-space photon wavelength calculated from Eq. (10) using the measured permittivity spectra. Our criterion for adequate SPP propagation length, ${ }^{8}$ $L_{z}>2 \lambda$, holds for the long-wave parts of the curve that lie above the dashed line. (The short-wave portions that lie above the dashed line apply to unbound waves, which nevertheless experience propagation loss due to interaction with the surface and thus have finite propagation lengths.) For carrier concentrations $1 \times 10^{20}$ and $6 \times 10^{19} \mathrm{~cm}^{-3}, L_{z}$ exceeds $2 \lambda$ for $\lambda>6.05$ and $16.6 \mu \mathrm{m}$, respectively.

\section{DISCUSSION}

Silicon plasmonic technology is part of a more general plasmo-photonic technology in which plasmonic waveguides couple seamlessly to photonic waveguides, and vice versa, in order to give higher performance and/or new functionality. Silicon plasmonics is a part of the group-IV plasmonics approach wherein the composite plasmonic waveguides feature the semiconductors $\mathrm{SiGe}, \mathrm{Ge}$, or $\mathrm{GeSn}$ (as well as $\mathrm{Si}$ )

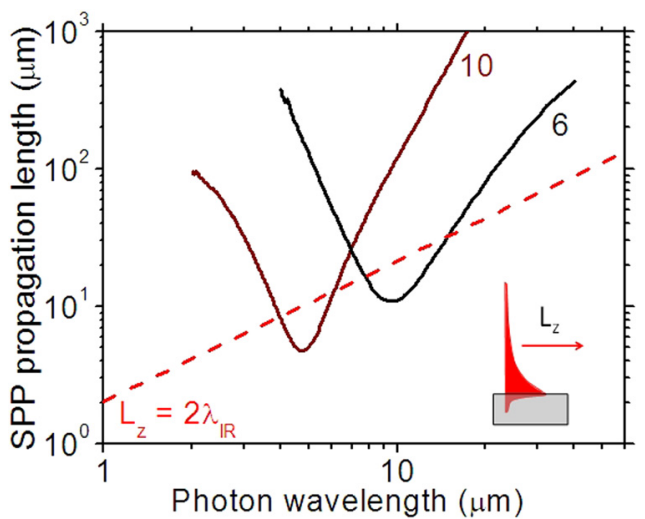

FIG. 6. (Color online) SPP energy propagation length vs free space wavelength. The region with $L_{z}>2 \lambda$ satisfies our criterion for the minimum useful propagation. The numbers that label the curves give the carrier concentrations $\left(\times 10^{19} \mathrm{~cm}^{-3}\right)$. The inset is a schematic suggesting the exponential decay of SPP intensity away from the interface and the significance of $L_{z}$. together with the novel doped-Ge and germanicide conductors (as well as doped-Si and silicides ${ }^{8}$ ). The realm of groupIV plasmonics is largely uncharted as yet and is an excellent subject for future study. This paper presents, for this emerging field, some initial experimental results that are of particular relevance to potential IR sensor applications.

For Si plasmonics, conductors of doped poly-silicon, ${ }^{22}$ and doped strained silicon ${ }^{23}$ are practical alternatives to doped crystalline Si. The strained versions are of particular interest due to enhanced mobilities. It is well known in the MOSFET industry that a layer of crystalline $\mathrm{Si}$ grown upon a relaxed layer of $\mathrm{Si}_{0.7} \mathrm{Ge}_{0.3}$ will result in a strained-Si layer whose electron and hole mobilities are increased by about 1.8 times. Higher mobility means longer relaxation time, which leads to lower loss for SPPs. The resulting smaller imaginary part of the permittivity would also give sharper SPP resonances than the ones observed here for bulk $\mathrm{Si}$, leading to better biosensor sensitivity.

\section{SUMMARY AND CONCLUSIONS}

Calculated and experimental SPP resonances at $10 \mu \mathrm{m}$ wavelengths were observed for $\mathrm{p}-\mathrm{Si}$ lamellar gratings with a carrier concentration of $1 \times 10^{20} \mathrm{~cm}^{-3}$. The resonances were distinct, and although rather broad, they may have value for sensing applications. Adequate mode confinement and propagation lengths, according to our criteria for useful SPP-based sensing, were found for this material when the excitation wavelength is between about 6 and $10 \mu \mathrm{m}$.

For material with a carrier concentration of $6 \times 10^{19}$ $\mathrm{cm}^{-3}$, no SPP resonance was observed since the plasma wavelength is longer than our available laser wavelengths. Calculations based on measured permittivity spectra indicate that for this material there was no wavelength where the criteria for both mode confinement and propagation could be satisfied simultaneously.

\section{ACKNOWLEDGMENTS}

This work was supported by the AFOSR (Gernot Pomrenke Program Manager) under Grant Nos. FA9550-1010030, FA9550-10-1-0417, and AFRL LRIR, Award Nos. 09RY09COR, 09RY01COR, and 00SN03COR, as well as by an NSF SBIR Phase I award IIP-0944520.

${ }^{1}$ S. A. Maier, Plasmonics: Fundamentals and Applications (Springer, New York, 2007).

${ }^{2}$ J. Homola, S. S. Yee, and G. Gauglitz, Sen. Actuator B-Chem. 54, 3 (1999).

${ }^{3}$ S. Patskovsky, A. V. Kabashin, M. Meunier, and J. H. T. Luong, J. Opt. Soc. Am. A 20, 1644 (2003).

${ }^{4}$ J. N. Yih, Y. M. Chu, Y. C. Mao, W. H. Wang, F. C. Chien, C. Y. Lin, K. L. Lee, P. K. Wei, and S. J. Chen, Appl. Opt. 45, 1938 (2006).

${ }^{5}$ J. W. Cleary, G. Medhi, R. E. Peale, W. Buchwald, O. Edwards, and I. Oladeji, Proc. SPIE 7673, 767306 (2010).

${ }^{6}$ R. Ziblat, V. Lirtsman, D. Davidov, and B. Aroeti, Biophys. J. 90, 2592 (2006).

${ }^{7}$ J. W. Cleary, R. E. Peale, D. Shelton, G. D. Boreman, R. Soref, W. Buchwald, Proc. Mat. Res. Soc. 1133, AA10 (2008).

${ }^{8}$ R. Soref, R. E. Peale, and W. Buchwald, Opt. Express 16, 6507 (2008).

${ }^{9}$ J. W. Cleary, R. E. Peale, D. J. Shelton, G. D. Boreman, C. W. Smith, M. Ishigami, R. Soref, A. Drehman, and W. R. Buchwald, J. Opt. Soc. Am. B 27, 730 (2010).

${ }^{10}$ S. Basu, B. J. Lee, and Z. Zhang, J. Heat Transfer 132, 023301 (2010). 
${ }^{11}$ Y.-B. Chen, Opt. Express 17, 3130 (2009).

${ }^{12}$ W. DiPippo, B. J. Lee, and K. Park, Opt. Express 18, 19396 (2010).

${ }^{13}$ J. C. Ginn, R. L. Jarecki, Jr., E. A. Shaner, and P. S. Davids, J. Appl. Phys. 110, 043110 (2011).

${ }^{14}$ M. Shahzad, G. Medhi, R. E. Peale, R. Tsuchikawa, M. Ishigami, W. Buchwald, J. Cleary, G. D. Boreman, O. Edwards, D. J. Diaz, and T. A. Gorman, Proc. SPIE 8024, 80240B (2011).

${ }^{15}$ J. W. Cleary, G. Medhi, R. E. Peale, and W. Buchwald, Appl. Optics 49, $3102(2010)$.

${ }^{16} \mathrm{H}$. Raether, Surface Plasmons on Smooth and Rough Surfaces and on Gratings (Springer-Verlag, Berlin, 1988).

${ }^{17}$ A. Hessel and A. A. Oliner, Appl. Opt. 4, 1275 (1965).
${ }^{18}$ S. M. Sze, Physics of Semiconductor Devices, $2^{\text {nd }}$ ed. (Wiley-Interscience, New York, 1981).

${ }^{19}$ H. G. Tompkins, A User's Guide to Ellipsometry (Academic, San Diego, 1993).

${ }^{20}$ E. B. Saff and A. D. Snider, Fundamentals of Complex Analysis with Applications to Engineering and Science, $3^{\text {rd }}$ ed. (Pearson Education, Upper Saddle River, NJ, 2003).

${ }^{21}$ L. D. Landau, E. M. Lifshitz, and L. P. Pitaevskii, Electrodynamics of Continuous Media, $2^{\text {nd }}$ ed. (Elsevier Butterworth Heinemann, Amsterdam, 1993), section 87.

${ }^{22}$ A. R. Brown, G. Roy, and A. Asenov, IEEE Trans. Electron Devices 54, 3056 (2007)

${ }^{23}$ M. V. Fischetti and S. E. Laux, J. Appl. Phys. 80, 2234 (1996). 